\title{
Assessment on Management of Yellow Mosaic Virus in Pole beans through Integrated Approach
}

\author{
B. Manjunath ${ }^{\text {** }}$, B. S. Rajendra Prasad ${ }^{2}$, S. Pavithra ${ }^{3}$, R. Manjunath ${ }^{4}$, \\ A. P. Mallikarjuna Gowda ${ }^{1}$, Savita S. Manganavar ${ }^{2}$, B. Gayathri ${ }^{4}$ and Y. D. Chithra ${ }^{1}$ \\ ${ }^{1}$ ICAR-Krishi Vigyan Kendra, Bengaluru Rural District, Karnataka, India \\ ${ }^{2}$ ICAR - Krishi Vigyan Kendra, Ramanagaram District, Karnataka, India \\ ${ }^{3}$ ICAR-Krishi Vigyan Kendra, Mandya District, Karnataka, India \\ ${ }^{4}$ ICAR- Krishi Vigyan Kendra, Chikkaballapura District, Karnataka, India \\ *Corresponding author
}

\section{A B S T R A C T}

\section{Keywords}

Pole bean, yellow mosaic virus, whiteflies, biocontrol agents, insecticides, management

\section{Article Info}

Accepted: 05 April 2020 Available Online: 10 May 2020
Pole bean, an important vegetable crop is becoming susceptible for yellow mosaic virus disease transmitted by whiteflies throughout the year. The present investigation was carried out to evaluate the efficacy of different integrated approaches for the management of the disease. Intercropping with two rows of border crops of maize 30 days before sowing of pole bean, mulching with black silver mulch, seed treatment with Thiomethaxam $25 \mathrm{WG}-5 \mathrm{~g} / \mathrm{kg}$ seeds during sowing, soil application of Pseudomonas fluorescens along with neem cake $(1 \mathrm{~kg} / 100 \mathrm{~kg})$, installation of yellow sticky trap @ 10 no/acre and spraying of seaweed extract $(1.5 \mathrm{ml} / \mathrm{L}) 20$ days after sowing, spraying of Thiamethoxam $25 \%$ WG $(0.5 \mathrm{~g} / \mathrm{L})$ and Imidacloprid $17.8 \mathrm{SL}(0.5 \mathrm{ml} / \mathrm{L}) 30$ and 45 days after sowing respectively recorded less disease incidence and higher yield compared to recommended practice.

\section{Introduction}

Pole bean (Phaseolus vulgaris $\mathrm{L}$ ) is a native of South America, where it has been cultivated as a staple food for centuries. The cultivars of French bean were bred to grow as vines which cling to poles or trellises, hence it is called pole bean. It is consumed as immature tender fruits, green grains as vegetables and dry grain (Rajmah). The nutritive value of $100 \mathrm{~g}$ of green pod contains $1.7 \mathrm{~g}$ protein, $0.1 \mathrm{~g}$ fat, $4.5 \mathrm{~g}$ carbohydrate, $1.8 \mathrm{~g}$ fibre and is also rich in minerals and vitamins. It has some medicinal properties in control of diabetes, cardiac problems and natural cure for bladder burn (Duke, 1981). 
Pole bean suffers from fungal, bacterial and viral diseases. Among them ascochyta blight, anthracnose, rust, root rot, angular leaf spot, bacterial blight, common bean mosaic virus, yellow mosaic virus and bean leaf roll virus. Among viral diseases yellow mosaic has been considered as an important limiting factor in pole bean productivity (Maramorosch and Muniyappa, 1981).

Yellow mosaic disease in pole bean is widely distributed in the tropical and sub-tropical regions. The symptom appears as brilliant yellow or golden yellow colour on leaves which may be partially or completely yellow. Cultivars express rugosity and rolling of leaves, while tolerant cultivars develop mild yellowing and often the plans show stunting. Pods exhibits blotching, discoloration with reduced size and number (Jyothi et al., 2013). The estimation of crop loss ranged from 40 to 100 per cent depending on the cultivar and time of infection (Pierre, 1975 and Galvez and Cardenas, 1980, Sharma et al., 2015).

Bemisia tabaci (Gennadius) (Aleyrodidae: Homoptera) is one of the important sucking pest which inflicts heavy damage to the crop, not only through direct loss of plant vitality by feeding cell sap but also by transmitting the yellow mosaic virus disease (Muniyappa, 1980).

Since pole bean is grown extensively throughout the year in and around Bengaluru rural district, Ramanagaram district, Mandya district and Chikkaballapura district as important vegetable crop, the crop succumb for many diseases. Moreover, the crop is becoming susceptible for yellow mosaic virus disease transmitted by whiteflies throughout the year.

The average yield of pole bean in the above mentioned districts is 25 t/ha as against potential yield of 35 t/ha which accounts for
$25 \%$ lesser yield. This is mainly due to the damage caused by yellow mosaic virus to the extent of $39 \%$ crop loss. In view of above facts, the on farm technology on management of yellow mosaic virus in pole bean through integrated approach was conducted in all the 4 districts in order to achieve higher yield, quality and profit.

\section{Materials and Methods}

The Krishnarajapura village, Doddaballapur, Bengaluru Rural district, Kalya village, Ramanagaram district, Nelamakanahalli village, Mandya district and Gadidasanahalli, Chikkaballapura district were selected for the case study, where in farmers were growing pole bean as main crop by following their own practices with indiscriminate use of pesticides with more cost of cultivation. The field experiment was carried out with four treatments with five replications. The treatment details are mentioned in Table 1.

The disease incidence observations were recorded from 20 days after sowing up to 60 days after sowing at an interval of 10 days and the data were analysed statistically. Yield data was recorded at different intervals of harvesting and the data was pooled at the final stage of the harvesting. Net returns from each treatment were calculated by considering the yield obtained and cost of treatment on hectare basis. Benefit: Cost (B: C ) ratio was calculated to compare the economic feasibility of various treatments.

\section{Results and Discussion}

The management of yellow mosaic virus in pole bean through integrated approach revealed that the per cent disease incidence of yellow mosaic virus across the districts was less at different days after sowing when compared to the recommended practice. The average disease incidence was less in 
alternate practice 2 compared to recommended practice in all the 4 districts (Table 1a, 2a, 3a and 4a) .The yield was recorded high in alternate practice 2 compared to recommended practice across all the 4 districts (Table 1b, 2b, 3b and 4b).

The combination of treatments in alternate practice 2 was found effective in managing the yellow mosaic disease in pole bean compared to recommended practice. The African tall maize sown 3 weeks before main crop acts as a barrier crop and restrict the movement of viruliferous whiteflies to get into the field and settle on the pole bean crop. These findings are in conformity with the findings as reported by Jyothi et al., (2013) and Jeevan (2013). Seed treatment with Thiomethaxam $25 \mathrm{WG}-5 \mathrm{~g} / \mathrm{kg}$ seeds can protect the young plants upto 30 to 40 days after sowing. Similar results were obtained by Jyothi et al., 2013 and Jeevan (2013). These results are in conformity with the findings as reported by Shankarappa (2002) in managing tomato leaf curl virus. Use of reflective mulches in between the rows will help in interfering on settlement of whiteflies on the host plant. The control of whiteflies is due to the fact that mulches reflects UV wavelength, which interfere with the orientation of whitefly and location on host (Charles Summer et al., 2005).

Soil application of Pseudomonas fluorescens along with neem cake $(1 \mathrm{~kg} / 100 \mathrm{~kg})$, installation of yellow sticky trap @ 10 no/acre and spraying of seaweed extract $(1.5 \mathrm{ml} / \mathrm{L}) 20$ days after sowing, spraying of Thiamethoxam $25 \% \mathrm{WG}(0.5 \mathrm{~g} / \mathrm{L})$ and Imidacloprid 17.8 SL $(0.5 \mathrm{ml} / \mathrm{L}) \quad 30$ and 45 days after sowing respectively recorded less disease incidence and higher yield compared to recommended practice (Table 5). Similar findings were reported by Panduranga et al., (2011) in managing mung bean yellow mosaic disease in green gram. The benefit cost ratio was found to be high in alternate practice 2 compared to recommended practice (Table 6).

Table.1a Percent disease incidence and yield of Pole beans in On Farm testing conducted on integrated management of yellow mosaic virus in pole beans during 2019-20 at Krishnarajapura,

Nelamangala Taluk, Bengaluru Rural District

\begin{tabular}{|c|c|c|c|c|c|c|c|}
\hline \multirow{2}{*}{$\begin{array}{c}\text { Treatment } \\
\text { details }\end{array}$} & \multicolumn{3}{|c|}{ Yellow mosaic virus incidence (\%) } & $\begin{array}{c}\text { Average } \\
\text { per cent } \\
\text { disease } \\
\text { incidence }\end{array}$ & $\begin{array}{c}\text { Yield } \\
\text { (t/ha) }\end{array}$ \\
\hline $\begin{array}{c}\text { Farmer's } \\
\text { Practice }\end{array}$ & 16.45 & 33.95 & 30.22 & 24.68 & 19.62 & 24.98 & 30.06 \\
\hline $\begin{array}{c}\text { Recommended } \\
\text { practice }\end{array}$ & 15.75 & 25.10 & 21.20 & 15.13 & 12.62 & 17.96 & 29.07 \\
\hline $\begin{array}{c}\text { Alternate } \\
\text { Practice 1 }\end{array}$ & 17.10 & 30.66 & 26.79 & 21.30 & 16.50 & 22.47 & 28.10 \\
\hline $\begin{array}{c}\text { Alternate } \\
\text { Practice 2 }\end{array}$ & 4.17 & 7.12 & 5.81 & 4.26 & 3.19 & 4.91 & 33.46 \\
\hline SEm $\mathbf{n}$ & 0.34 & 0.73 & 0.59 & 0.46 & 0.32 & 0.49 & 1.00 \\
\hline CD (0.05) & 1.12 & 2.39 & 1.92 & 1.51 & 1.03 & 1.59 & 3.25 \\
\hline CV & 7.27 & 7.45 & 7.33 & 7.36 & 7.53 & 7.39 & 7.14 \\
\hline
\end{tabular}

DAS - Days After Sowing 
Table.1b Economics of treatments evaluated for the management of yellow mosaic virus in pole beans during 2019-20

\begin{tabular}{|l|l|l|l|l|l|l|c|}
\hline Treatments & $\begin{array}{l}\text { Yield } \\
\text { (t/ha) }\end{array}$ & $\begin{array}{l}\text { No. of } \\
\text { sprays }\end{array}$ & $\begin{array}{l}\text { Cost of } \\
\text { sprays } \\
\text { (Rs./ha) }\end{array}$ & $\begin{array}{l}\text { Cost of } \\
\text { cultivation/ } \\
\text { ha }\end{array}$ & $\begin{array}{l}\text { Gross } \\
\text { returns }\end{array}$ & $\begin{array}{l}\text { Net } \\
\text { returns }\end{array}$ & $\begin{array}{l}\text { B:C } \\
\text { ratio }\end{array}$ \\
\hline T1 (FP) & 29.78 & 05 & 18921 & 149133 & 573866 & 424733 & 3.85 \\
\hline T2(RP) & 28.62 & 02 & 7900 & 139781 & 583733 & 443951 & 4.17 \\
\hline T3(AP1) & 27.86 & 02 & 6150 & 142150 & 564866 & 422716 & 3.97 \\
\hline T4(AP2) & $\mathbf{3 3 . 1 1}$ & $\mathbf{0 3}$ & $\mathbf{1 1 7 9 5}$ & $\mathbf{1 4 5 2 7 0}$ & $\mathbf{7 0 8 5 4 6}$ & $\mathbf{5 6 3 2 7 6}$ & $\mathbf{4 . 8 8}$ \\
\hline
\end{tabular}

FP - Farmers practice, RP - Recommended practice, AP1 -Alternate practice 1, AP2 - Alternate practice 2

Table.2a Percent disease incidence and yield of Pole beans in On Farm testing conducted on integrated management of yellow mosaic virus in pole beans during 2019-20 at Kalya, Magadi Taluk, Ramanagaram District

\begin{tabular}{|c|c|c|c|c|c|c|c|}
\hline \multirow{2}{*}{$\begin{array}{c}\text { Treatment } \\
\text { details }\end{array}$} & \multicolumn{3}{|c|}{ Yellow mosaic virus incidence (\%) } & $\begin{array}{l}\text { Average per } \\
\text { cent disease } \\
\text { incidence }\end{array}$ & $\begin{array}{l}\text { Yield } \\
\text { (t/ha) }\end{array}$ \\
\hline $\begin{array}{c}\text { Farmer's } \\
\text { Practice }\end{array}$ & 17.10 & 30.66 & 26.79 & 21.30 & 16.50 & 22.47 & 29.25 \\
\hline $\begin{array}{c}\text { Recommended } \\
\text { practice }\end{array}$ & 10.95 & 25.10 & 20.07 & 16.62 & 10.15 & 16.58 & 28.27 \\
\hline $\begin{array}{c}\text { Alternate } \\
\text { Practice 1 }\end{array}$ & 17.24 & 27.93 & 24.50 & 17.60 & 13.40 & 20.13 & 27.50 \\
\hline $\begin{array}{c}\text { Alternate } \\
\text { Practice 2 }\end{array}$ & 6.72 & 9.41 & 7.28 & 5.88 & 3.44 & 6.55 & 32.76 \\
\hline SEm + & 0.39 & 0.74 & 0.53 & 0.40 & 0.36 & 0.48 & 1.12 \\
\hline CD (0.05) & 1.26 & 2.43 & 1.72 & 1.31 & 1.16 & 1.58 & 3.65 \\
\hline CV & 6.54 & 7.99 & 6.70 & 6.98 & 8.11 & 7.26 & 8.22 \\
\hline
\end{tabular}

DAS - Days After Sowing

Table.2b Economics of treatments evaluated for the management of yellow mosaic virus in pole beans during 2019-20

\begin{tabular}{|l|l|l|l|l|l|l|c|}
\hline Treatments & $\begin{array}{l}\text { Yield } \\
\text { (t/ha) }\end{array}$ & $\begin{array}{l}\text { No. of } \\
\text { sprays }\end{array}$ & $\begin{array}{l}\text { Cost of } \\
\text { sprays } \\
\text { (Rs./ha) }\end{array}$ & $\begin{array}{l}\text { Cost of } \\
\text { cultivation/ } \\
\text { ha }\end{array}$ & $\begin{array}{l}\text { Gross } \\
\text { returns }\end{array}$ & $\begin{array}{l}\text { Net } \\
\text { returns }\end{array}$ & B:C ratio \\
\hline T1 (FP) & 29.78 & 05 & 18600 & 143219 & 585000 & 441781 & 4.08 \\
\hline T2(RP) & 28.62 & 02 & 7600 & 139246 & 565400 & 426154 & 4.06 \\
\hline T3(AP1) & 27.86 & 02 & 5900 & 142700 & 550000 & 407300 & 3.85 \\
\hline T4(AP2) & $\mathbf{3 3 . 1 1}$ & $\mathbf{0 3}$ & $\mathbf{1 1 0 4 5}$ & $\mathbf{1 4 4 6 9 0}$ & $\mathbf{6 8 7 9 6 0}$ & $\mathbf{5 4 3 2 7 0}$ & $\mathbf{4 . 7 5}$ \\
\hline
\end{tabular}

FP - Farmers practice, RP - Recommended practice, AP1 -Alternate practice 1,

AP2 - Alternate practice 2 
Table.3a Percent disease incidence and yield of Pole beans in On Farm testing conducted on integrated management of yellow mosaic virus in pole beans during 2019-20 at Nelamakanahalli,

Malavalli Taluk, Mandya District

\begin{tabular}{|c|c|c|c|c|c|c|c|}
\hline \multirow{2}{*}{ Treatment details } & \multicolumn{5}{|c|}{ Yellow mosaic virus incidence (\%) } & $\begin{array}{c}\text { Average per } \\
\text { cent disease } \\
\text { incidence }\end{array}$ & $\begin{array}{l}\text { Yield } \\
\text { (t/ha) }\end{array}$ \\
\cline { 2 - 8 } & 20 DAS & 30 DAS & 40 DAS & 50 DAS & 60 DAS & \\
\hline Farmer's Practice & 16.45 & 33.95 & 30.22 & 24.68 & 19.62 & 24.98 & 29.64 \\
\hline Recommended practice & 14.95 & 21.10 & 24.07 & 16.62 & 10.15 & 17.38 & 28.65 \\
\hline Alternate Practice 1 & 15.17 & 28.31 & 24.50 & 19.60 & 14.12 & 20.34 & 27.97 \\
\hline Alternate Practice 2 & 5.47 & 8.47 & 6.20 & 4.86 & 3.91 & 5.78 & 33.06 \\
\hline SEm \pm & 0.34 & 0.73 & 0.59 & 0.46 & 0.32 & 0.49 & 0.95 \\
\hline CD (0.05) & 1.12 & 2.39 & 1.92 & 1.51 & 1.03 & 1.59 & 3.09 \\
\hline CV & 7.27 & 7.45 & 7.33 & 7.36 & 7.53 & 7.39 & 6.86 \\
\hline
\end{tabular}

DAS - Days After Sowing

Table.3b Economics of treatments evaluated for the management of yellow mosaic virus in pole beans during 2019-20

\begin{tabular}{|l|c|c|c|c|c|c|c|}
\hline Treatments & $\begin{array}{l}\text { Yield } \\
\text { (t/ha) }\end{array}$ & $\begin{array}{l}\text { No. of } \\
\text { sprays }\end{array}$ & $\begin{array}{l}\text { Cost of } \\
\text { sprays } \\
\text { (Rs./ha) }\end{array}$ & $\begin{array}{l}\text { Cost of } \\
\text { cultivation/ } \\
\text { ha }\end{array}$ & $\begin{array}{l}\text { Gross } \\
\text { returns }\end{array}$ & $\begin{array}{l}\text { Net } \\
\text { returns }\end{array}$ & B:C ratio \\
\hline T1 (FP) & 29.78 & 05 & 18150 & 144600 & 563160 & 418560 & 3.89 \\
\hline T2(RP) & 28.62 & 02 & 7450 & 142750 & 544350 & 401600 & 3.81 \\
\hline T3(AP1) & 27.86 & 02 & 6000 & 143500 & 531430 & 387930 & 3.70 \\
\hline T4(AP2) & $\mathbf{3 3 . 1 1}$ & $\mathbf{0 3}$ & $\mathbf{1 1 2 0 0}$ & $\mathbf{1 4 5 2 0 0}$ & $\mathbf{6 9 4 2 6 0}$ & $\mathbf{5 4 9 0 6 0}$ & $\mathbf{4 . 7 8}$ \\
\hline
\end{tabular}

FP - Farmers practice, RP - Recommended practice, AP1 -Alternate practice 1,

AP2 - Alternate practice 2

Table.4a Percent disease incidence and yield of Pole beans in On Farm testing conducted on integrated management of yellow mosaic virus in pole beans during 2019-20 at Gadidasanahalli,

Chintamani Taluk, Chikkaballapura District

\begin{tabular}{|c|c|c|c|c|c|c|c|}
\hline Treatment details & \multicolumn{5}{|c|}{ Yellow mosaic virus incidence (\%) } & $\begin{array}{c}\text { Average per } \\
\text { cent disease } \\
\text { incidence }\end{array}$ & $\begin{array}{l}\text { Yield } \\
\text { (t/ha) }\end{array}$ \\
\cline { 2 - 9 } & 20 DAS & 30 DAS & 40 DAS & 50 DAS & 60 DAS & \\
\hline Farmer's Practice & 14.30 & 30.20 & 26.10 & 21.22 & 17.82 & $\begin{array}{c}21.93 \\
(27.73)\end{array}$ & 30.15 \\
\hline $\begin{array}{c}\text { Recommended } \\
\text { practice }\end{array}$ & 10.95 & 25.10 & 20.07 & 16.62 & 10.15 & 16.58 & 28.47 \\
\hline Alternate Practice 1 & 16.25 & 28.02 & 22.62 & 15.29 & 11.46 & 18.73 & 27.85 \\
\hline Alternate Practice 2 & 6.17 & 8.67 & 7.81 & 5.26 & 4.19 & 6.42 & 33.16 \\
\hline SEm \pm & 0.34 & 0.73 & 0.59 & 0.46 & 0.32 & 0.49 & 0.95 \\
\hline CD (0.05) & 1.12 & 2.39 & 1.92 & 1.51 & 1.03 & 1.59 & 3.33 \\
\hline CV & 7.27 & 7.45 & 7.33 & 7.36 & 7.53 & 7.39 & 7.37 \\
\hline
\end{tabular}

DAS - Days after Sowing 
Table.4b Economics of treatments evaluated for the management of yellow mosaic virus in pole beans during 2019-20

\begin{tabular}{|l|c|c|c|c|l|l|l|}
\hline Treatments & $\begin{array}{l}\text { Yield } \\
\text { (t/ha) }\end{array}$ & $\begin{array}{l}\text { No. of } \\
\text { sprays }\end{array}$ & $\begin{array}{l}\text { Cost of } \\
\text { sprays } \\
\text { (Rs./ha) }\end{array}$ & $\begin{array}{l}\text { Cost of } \\
\text { cultivation// } \\
\text { ha }\end{array}$ & $\begin{array}{l}\text { Gross } \\
\text { returns }\end{array}$ & $\begin{array}{l}\text { Net } \\
\text { returns }\end{array}$ & $\begin{array}{l}\text { B:C } \\
\text { ratio }\end{array}$ \\
\hline T1 (FP) & 29.78 & 05 & 18650 & 146520 & 603000 & 456480 & 4.11 \\
\hline T2(RP) & 28.62 & 02 & 7700 & 140290 & 569400 & 429110 & 4.05 \\
\hline T3(AP1) & 27.86 & 02 & 5950 & 142150 & 557000 & 414850 & 3.92 \\
\hline T4(AP2) & $\mathbf{3 3 . 1 1}$ & $\mathbf{0 3}$ & $\mathbf{1 1 5 0 0}$ & $\mathbf{1 4 6 1 0 0}$ & $\mathbf{6 9 6 3 6 0}$ & $\mathbf{5 5 0 2 6 0}$ & $\mathbf{4 . 7 7}$ \\
\hline
\end{tabular}

FP - Farmers practice, RP - Recommended practice, AP1 -Alternate practice 1, AP2 - Alternate practice 2

Table.5 Pooled data of percent disease incidence and yield of Pole beans in On Farm Testing conducted on integrated management of yellow mosaic virus in pole beans at different regions

\begin{tabular}{|c|c|c|c|c|c|c|c|c|c|c|}
\hline \multirow{4}{*}{$\begin{array}{c}\text { Treatmen } \\
\mathrm{t} \text { details }\end{array}$} & \multicolumn{8}{|c|}{ Average Per cent Disease Incidence } & \multirow{4}{*}{$\begin{array}{l}\text { Poole } \\
\text { d PDI }\end{array}$} & \multirow{4}{*}{$\begin{array}{c}\text { Pooled } \\
\text { yield }\end{array}$} \\
\hline & \multirow{2}{*}{\multicolumn{2}{|c|}{\begin{tabular}{|l|}
\multicolumn{2}{|c|}{$2019-20$} \\
Krishnarajapura, \\
Nelamangala Taluk, \\
Bengaluru Rural \\
District
\end{tabular}}} & \multirow{2}{*}{\multicolumn{2}{|c|}{\begin{tabular}{|l|}
\multicolumn{1}{c|}{$2019-20$} \\
Kalya, Magadi \\
Taluk, \\
Ramanagaram \\
District
\end{tabular}}} & \multirow{2}{*}{\multicolumn{2}{|c|}{\begin{tabular}{l}
\multicolumn{1}{c}{$2019-20$} \\
Nelamakanahal \\
li, Malavalli \\
Taluk, Mandya \\
District
\end{tabular}}} & \multirow{2}{*}{\multicolumn{2}{|c|}{\begin{tabular}{l}
\multicolumn{1}{c}{$2019-20$} \\
Gadidasanahalli, \\
Chintamani \\
Taluk, \\
Chikkaballapura \\
District
\end{tabular}}} & & \\
\hline & & & & & & & & & & \\
\hline & PDI & $\begin{array}{l}\text { Yield } \\
\text { (t/ha) }\end{array}$ & PDI & $\begin{array}{l}\text { Yield } \\
\text { (t/ha) }\end{array}$ & PDI & $\begin{array}{l}\text { Yield } \\
\text { (t/ha) }\end{array}$ & PDI & $\begin{array}{l}\text { Yield } \\
\text { (t/ha) }\end{array}$ & & \\
\hline $\begin{array}{l}\text { Farmer's } \\
\text { Practice }\end{array}$ & 24.98 & 30.06 & 22.47 & 29.25 & 24.98 & 29.64 & 21.93 & 30.15 & 23.59 & 29.78 \\
\hline $\begin{array}{l}\text { Recomme } \\
\text { nded } \\
\text { practice }\end{array}$ & 17.96 & 29.07 & 16.58 & 28.27 & 17.38 & 28.65 & 16.58 & 28.47 & 17.13 & 28.62 \\
\hline $\begin{array}{l}\text { Alternate } \\
\text { Practice } 1\end{array}$ & 22.47 & 28.10 & 20.13 & 27.50 & 20.34 & 27.97 & 18.73 & 27.85 & 20.42 & 27.86 \\
\hline $\begin{array}{l}\text { Alternate } \\
\text { Practice } 2\end{array}$ & 4.91 & 33.46 & 6.55 & 32.76 & 5.78 & 33.06 & 6.42 & 33.16 & 5.92 & 33.11 \\
\hline SEm \pm & 0.49 & 1.00 & 0.48 & 1.12 & 0.49 & 0.95 & 0.49 & 0.95 & 0.49 & 1.00 \\
\hline $\begin{array}{c}\text { CD at } \\
5 \%\end{array}$ & 1.59 & 3.25 & 1.58 & 3.65 & 1.59 & 3.09 & 1.59 & 3.33 & 1.59 & 3.33 \\
\hline CV & 7.39 & 7.14 & 7.26 & 8.22 & 7.39 & 6.86 & 7.39 & 7.37 & 7.36 & 7.40 \\
\hline
\end{tabular}


Table.6 Economics of treatments evaluated for the management of yellow mosaic virus in pole beans

\begin{tabular}{|l|c|c|c|c|c|c|c|}
\hline Treatments & $\begin{array}{c}\text { Yield } \\
\text { (t/ha) }\end{array}$ & $\begin{array}{c}\text { No. of } \\
\text { sprays }\end{array}$ & $\begin{array}{c}\text { Cost of } \\
\text { sprays } \\
\text { (Rs./ha) }\end{array}$ & $\begin{array}{c}\text { Cost of } \\
\text { cultivation/ } \\
\text { ha }\end{array}$ & $\begin{array}{c}\text { Gross } \\
\text { returns }\end{array}$ & $\begin{array}{c}\text { Net } \\
\text { returns }\end{array}$ & B:C ratio \\
\hline T1 (FP) & 29.78 & 05 & 18580 & 145868 & 581256 & 435388 & 3.98 \\
\hline T2(RP) & 28.62 & 02 & 7663 & 140516 & 565720 & 425203 & 4.02 \\
\hline T3(AP1) & 27.86 & 02 & 6000 & 142625 & 550824 & 408199 & 3.86 \\
\hline T4(AP2) & $\mathbf{3 3 . 1 1}$ & $\mathbf{0 3}$ & $\mathbf{1 1 3 8 5}$ & $\mathbf{1 4 5 3 1 5}$ & $\mathbf{6 9 6 7 8 1}$ & $\mathbf{5 5 1 4 6 6}$ & $\mathbf{4 . 8 0}$ \\
\hline
\end{tabular}

Table.7 Schedule of technology application for the management of yellow mosaic virus in pole beans

\begin{tabular}{|c|c|c|}
\hline Time of application & Chemical/Product & Quantity/dosage \\
\hline Before sowing & $\begin{array}{l}\text { Intercropping with two rows of } \\
\text { border crops of maize days before } \\
\text { sowing } \\
\text { Soil application of Pseudomonas } \\
\text { fluorescens along with neem cake }\end{array}$ & $\begin{array}{l}\text { (1 kg Pseudomonas fluorescens } \\
\text { in } 100 \mathrm{~kg} \text { neem cake) }\end{array}$ \\
\hline Sowing time & $\begin{array}{l}\text { Seed treatment with } \\
\text { Thiomethaxam } 25 \mathrm{WG}-5 \mathrm{~g} / \mathrm{kg} \\
\text { seeds, Mulching with black silver } \\
\text { mulch }\end{array}$ & $5 \mathrm{~g} / \mathrm{Kg}$ seeds \\
\hline 20 Days After Sowing & $\begin{array}{l}\text { Spraying of seaweed extract } \\
\text { Installation of yellow sticky trap }\end{array}$ & $\begin{array}{l}1.5 \mathrm{ml} / 1 \text { itre of water } \\
10 \mathrm{no} / \text { acre }\end{array}$ \\
\hline 30 Days After Sowing & Thiamethoxam $25 \% \mathrm{WG}$ & $0.5 \mathrm{~g} /$ litre of water \\
\hline 45 Days After Sowing & Imidacloprid 17.8 SL & $0.5 \mathrm{ml} /$ litre of water \\
\hline
\end{tabular}

Based on the present work, schedule of technology application for effective and efficient management of yellow mosaic virus in pole beans has been developed and also residue free produce can be obtained (Table 7). This schedule found to be more ecofriendly, environmentally compatible and safe for human health as well as agro-ecosystem.

Majority of the pole bean growing farmers are using pesticides indiscriminately would increase the cost of production and resurgence in the vector. The integrated approaches like growing border crop, use of reflective mulches and recommended dose of chemicals for management of the vector would not only reduces the cost of production but also reduces the disease by increasing the yield.

\section{Acknowledgement}

Authors are thankful to Agricultural Technology Application Research Institute (ATARI), Indian Council of Agricultural Research, Zone XI India for their kind guidance, motivation and financial support for this work.

\section{References}

Charles G Summer, Jeffrey P. Mitchell and James J Stapleton. (2005). Mulches 
reduce aphid borne viruses and whiteflies in cantaloupe. California Agric., 59: 90-94.

Duke, J.A. (1981). Handbook of legumes of world economic importance. pp 195200, New York, USA/London, UK: Plenum press.

Galvez, G.E. and Cardenas, M. (1980). Whitefly transmitted viruses. In: "Bean production problems" (eds. H.F. Schwartz and G.E.Galvez) pp 261-289, Centro International de Agricultura Tropica, Cali, Columbia.

Jeevan, B. (2013). Molecular characterization of yellow mosaic virus in pole bean (Phaseolus vulgaris L.) and its disease management. M.Sc. (Agri.) Thesis, Univ. Agri. Sci. Bangalore, Karnataka, $131 \mathrm{pp}$.

Jyothi, V., Nagaraju, N., Padmaja, A.S. and Rangaswamy, K.T. (2013). Transmission, detection and management of yellow mosaic virus in pole bean (Phaseolus vulgaris L.) J. Mycol. Plant Pathol., 43(2): 229-236.

Maramorosch, K. and Muniyappa, V. (1981). Whitefly transmitted plant disease agents in Karnataka, India. In: International workshop on pathogens transmitted by whiteflies. July 31,
(Oxford: Association of Applied Biologist, Wellesbourne).

Muniyappa, V. (1980). Whiteflies, In: Vectors of Plant Pathogens, K.F. Harris and K. Maramorosch (Eds),, pp 39-85, Academic Press, New York, 184 pp.

Panduranga, G.S., Vijayalakshmi, K. and Reddy L. Loka. (2011). Evaluation of insecticides for management of Bemisia tabaci and Mymv disease in Mung bean [Vigna radiata (L.) Wilczek]. Annals of Plant Protection Sciences. 19(2): 295298.

Pierre, R.E. (1975). Observation on golden mosaic of bean (Phaseolus vulgaris L.) in Jamaica. In: "Tropical diseases of legumes" (J. Bird and K. Maramorosch) pp 35-39.

Shankarappa, K.S. (2002). Spread of Tomato leaf curl virus and Bemisia tabaci Genn. in open pollinated tomato varieties. M.Sc. (Agri.) Thesis, Univ. Agri. Sci. Bangalore, Karnataka, 96 pp.

Sharma, P.N., Vivek Sharma, Anuradha Sharma, Kajal Rajput and Sharma, S.K. (2015). Identification and molecular characterization of bean yellow mosaic virus infecting french bean in Himachal Pradesh. Virus Disease, 26: 315-318.

\section{How to cite this article:}

Manjunath. B., B. S. Rajendra Prasad, S. Pavithra, R. Manjunath, A. P. Mallikarjuna Gowda, Savita S. Manganavar, B. Gayathri and Chithra. Y. D. 2020. Assessment on Management of Yellow Mosaic Virus in Pole beans through Integrated Approach. Int.J.Curr.Microbiol.App.Sci. 9(05): 172-179. doi: https://doi.org/10.20546/ijcmas.2020.905.019 